\title{
Design of a Distribution Management System for IPTV Systems
}

\author{
Jaegeol Yim, Gyeyoung Lee and Kyubark Shim \\ Dept. of Computer Engineering, Dongguk University at Gyeongju, \\ Gyeongbuk Korea \\ \{yim,lky,shim\}@dongguk.ac.kr
}

\begin{abstract}
IPTV, acronym of Internet Protocol Television, is a system that delivers teteyision services through Internet protocol networks. Therefore, an IPTV system consists of many subsystems including content management, user management, and broladcast management systems. Among the main subsystems, the distribution management system handles purchasing orders from distributors, transcoding original content into the format designated by the distributor, and transmitting transcoded content to the server designated by the distributor. In order to realize one source multi-use and to boost content circulation, an IPTV system should be open in that any authorized content providers are allowed to upload their content for circulation and any authorized distributors are allowed to circulate content. The distribution management subsystem of an IPTV system allows distributors to request for content circulation and system managers to proeess distributors' requests. Therefore, a distribution management system is one of the host important sybsystems of an IPTV system. This paper introduces our design of a distribution management system for IPTV systems.
\end{abstract}

Keywords: IPTV, Distibytion Manougement System, Content Management System, User Management System, One Source Malti-Use.

\section{Introduction}

One of the most successful examples of convergence is the Internet Protocol Television (IPTV) system, which is a convergence of communication and broadcast methods. IPTV is defined by the ITU-T FG IPTV as: "IPTV is defined as multimedia services such as television/video/audio/text/graphics/data delivered over IP based networks managed to provide the required level of quality of service and experience, security, interactivity and reliability" $[1,2]$.

There are many IPTV system configurations proposed by various organizations. ZooinNet Co. proposed a configuration of centralized IPTV system consisting of Broadcasting center, IDC (Internet Data Center), and Branch offices [3]. The authors of [4] introduced their research results to reduce network traffic. The authors of [5] introduced a simulation tool with which we can estimate network traffic of IPTV systems accurately.

However, there are few IPTV systems in which content can be purchased for distribution. The authors of [6] proposed an IPTV system that consists of many subsystems such as a content registration system, a distribution management system, a metadata hub system, a global interface system, a user management system, an operation log system, a distribution log system, a deploy system, and an authoring system. This system allows content distributors to purchase content to circulate. One of the main subsystems that are essential for circulating content is distribution 
management system. This paper introduces our design of a distribution management system.

\section{Related works}

The authors of [6] introduced a design of an IPTV system as shown in Figure 1. The system consists of many subsystems. Each of them requires computer servers. The hardware structure of the system is shown in Figure 1.

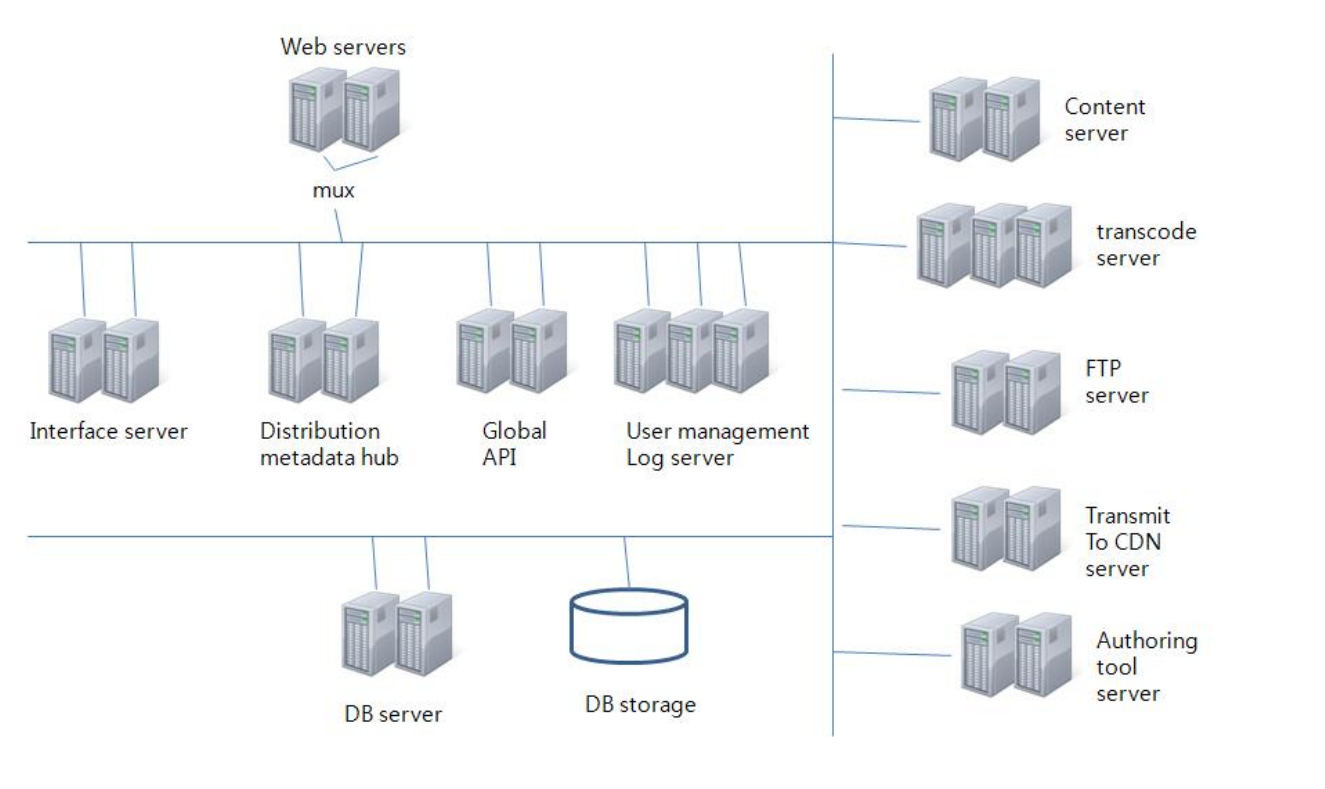

Figure 1. Description of the Hardware Structure of the IPTV System [6]

1) 2 web application servers for content registration: Windows Server 2008, IIS 7.0, Kendo UI control, content registration web application, open Flash Player, and the content registration Web application are installed here.

2) 2 interface servers: Windows Server 2008, IIS 7.0 and the content registration web service progran are installed here.

3) 2 servers for distribution metadata hub: Cent OS 6.3, Spring 3.1, Tomcat 7.0, JAVA, distribution meta hub web application program are installed here.

4) 2 global API servers: Cent OS 6.3, Spring 3.1, Tomcat 7.0, JAVA, global API interface program are installed here.

5) 3 servers for user management and log: Cent OS 6.3, Spring, Tomcat, MySQL, JAVA, the distribution log system, the operation log system, and the user management system are installed here.

6) 2 content essence hub servers: These servers allow users to store, retrieve, manage essence files. Cent OS 6.0, Spring 3.2, Gson 1.4, Tomcat, Apache, cronolog are installed here.

7) 3 transcode servers: ffmpeg, faac, x264-0.128, yasm-1.2.0, nodejs-0.8.19, curl-7.19.7, tinyxml2-1.0.9, jconcpp-0.6.0 rc2 are installed here.

8) 2 FTP servers: Cent OS, vftpd, and a Wowza Media Server are installed here. 
9) 2 servers for transmitting to CDN: Cent OS, nodejs, curl, tinyxml, jconcpp are installed here.

10) 2 authoring tool servers: Windows 7 , .Net framework, Declink drivers are installed here.

11) 2 cross media DB servers: Cent OS and Oracle $11 \mathrm{~g}$ are installed.

12) 1 storage: A huge capacity storage to store information of content providers, metadata for essence files, metadata for distribution, and essence files.

A database system for IPTV systems was introduced in [7]. A fast algorithm for IPTV system response time analysis was introduced in [8]. A protocol for secure mobile XPTV service delegation to support service level agreements was proposed in [9]. A chapnel domain system that logically extends channel allocation capability over limited channel resources was proposed in [10]. A handover scheme over standard Mobile IPY 6 that provide good quality of service was proposed in [11].

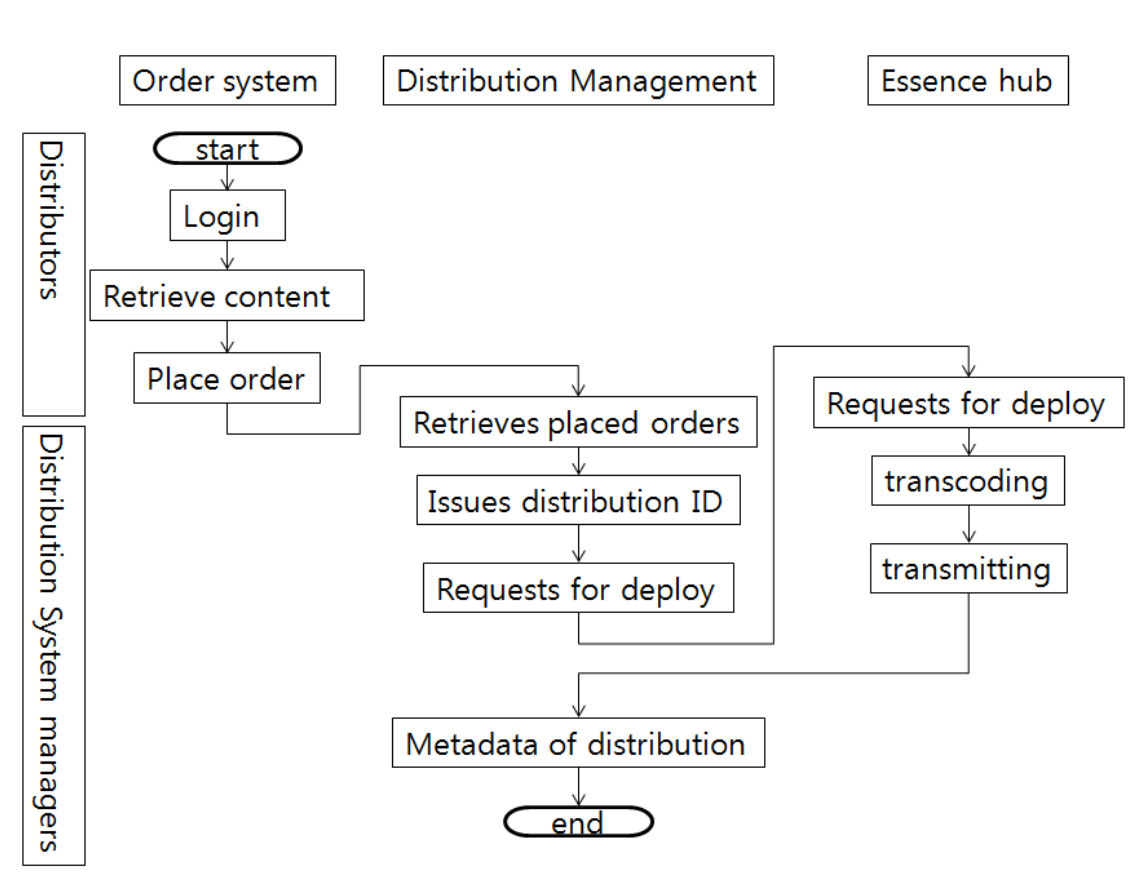

\section{Figure 2 The Process of Issuing Distribution ID and Recording Metadata of Distribution}

\section{Design of the System}

\subsection{Functional Requirements}

The distribution management system allows distributors to circulate content in the IPTV system by interacting with other component systems of the IPTV system. As is shown in Figure 2, the distribution management system allows the system managers to retrieve placed orders, to accept or reject orders, to issue distribution IDs for all accepted orders, and to request the essence hub system to deploy the ordered content. This system allows system managers to record metadata of the distribution when a deployment completion message arrives. 
Distribution management system records all events of issuing distribution IDs, of writing metadata of distribution, and of requesting for deployment in the Log System as shown in Figure 3.

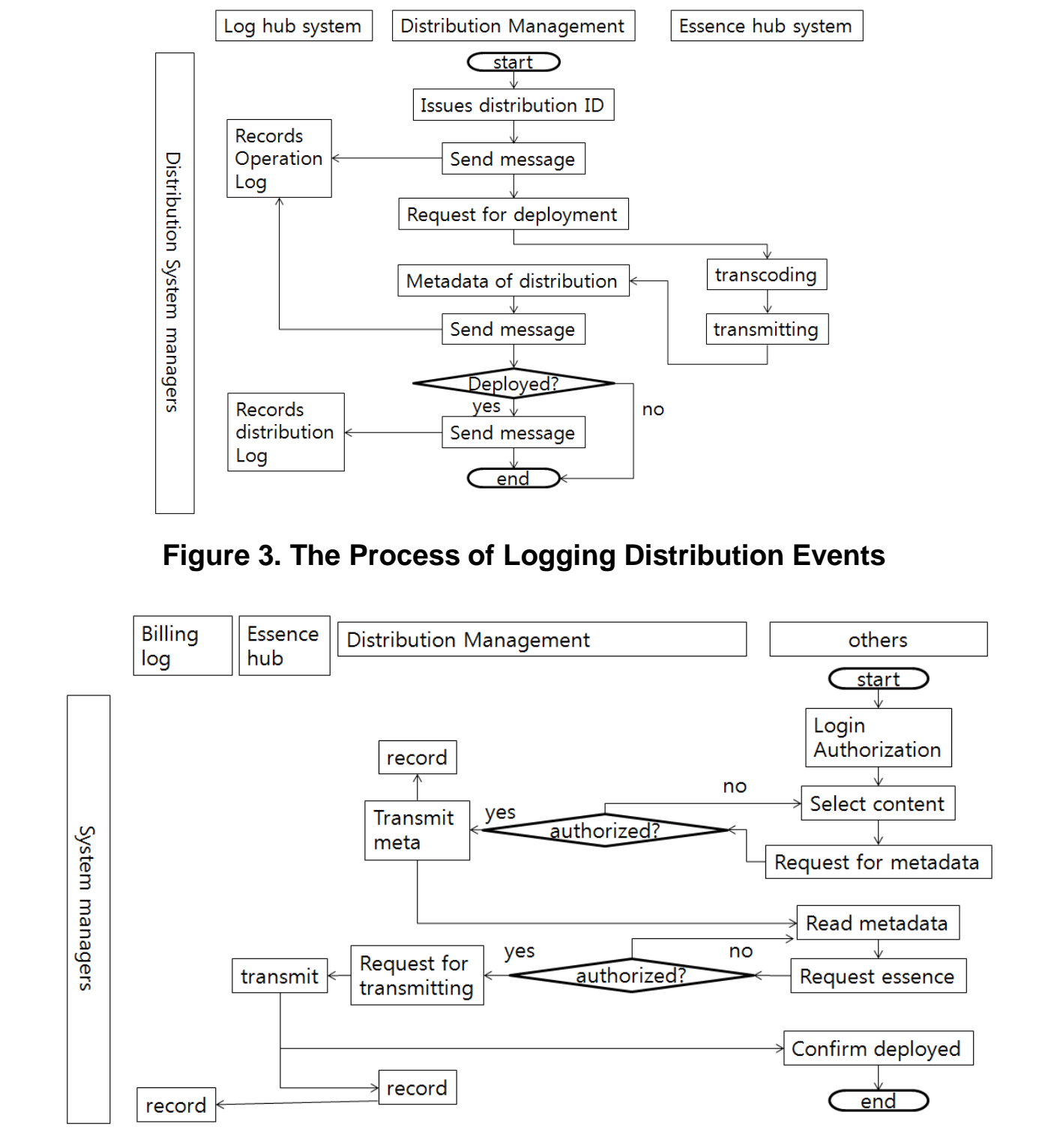

Figure 4. The Process of Using Distribution Metadata

Authenticated users can select content for distribution and request for metadata of the selected content. Then the distribution management system checks if the user is authorized to use the content. If the test is positive then the distribution management system transmits the requested metadata to the user and records the event of deploying the metadata. Referring to the metadata, the user can request for content for distribution. After checking authority, the distribution management system asks the essence hub to send the content for distribution to the user. The event of sending the content for distribution out is recorded in the operation log system and the billing log system. 


\subsection{User Interface}

This system allows system managers to retrieve placed orders, to select orders, and accept or reject selected orders. Therefore, we need a user interface as shown in Figure 5. In the figure, we can find a list of all content requested for distribution. We can specify a distributor name or a content group as search condition. After making check marks on interested items, we can click the button, 'approve' or 'reject'. If the manager clicks the button, 'approve', the system pops up a window in which a confirmation button is shown, whereas if the manager clicks the reject button, then the system pops up a window where the manager can describe the reason of rejection. By selecting the menu 'Approved'/'Rejected', we can retrieve information of approved/rejected purchase orders.
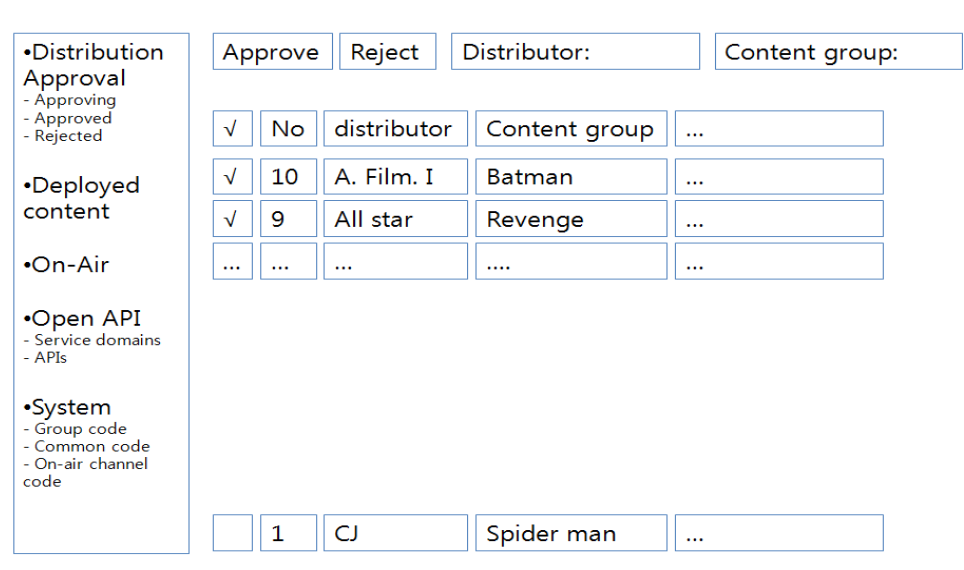

\section{Figure 5. An Example User Interface for Approving Purchase Orders}

The menu, 'Deployed content', allows the user to retrieve a list of deployed content as shown in Figure 6. We can specify distribution ID, content name and/or a period of time as the search condition. By selecting a distribution ID on this screen, system managers can type in metadata of the distributed content. Metadata can be classified into standard distribution metadata, deployment information, and essence file metadata. Standard distribution metadata can be further classified into basic attributes, KEM (Korea Educational Metadata) and PPL (Product placement) Figure 7 shows an example UI for typing in basic distribution metadata.

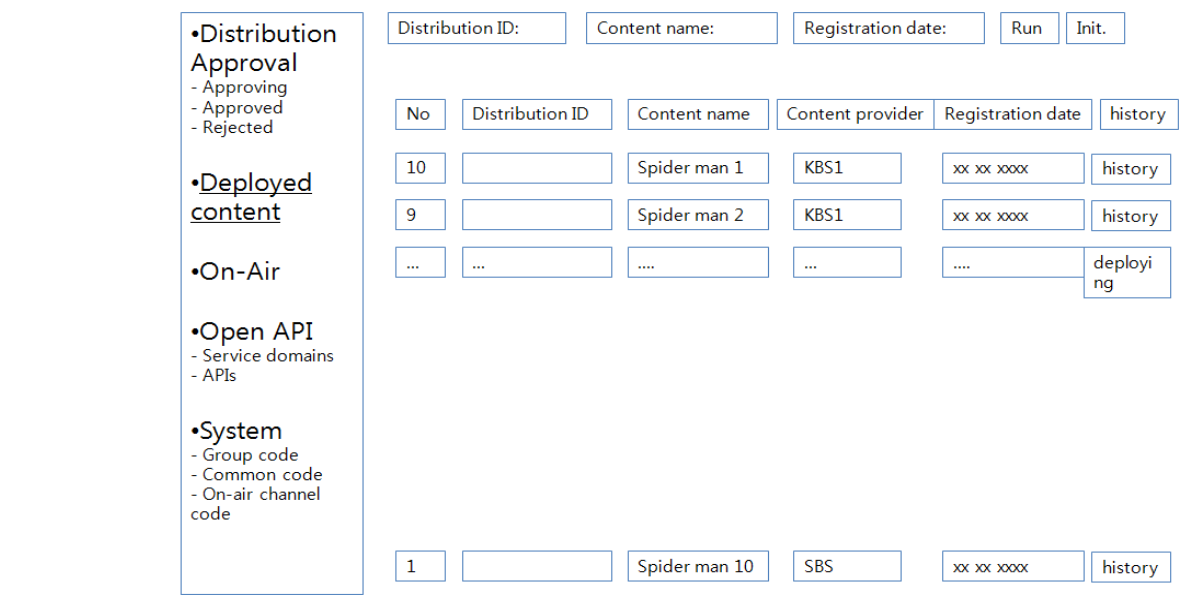

Figure 6. Our User Interface for 'Deployed Content' 


\begin{tabular}{|c|c|}
\hline Basic attributes & KEM attribu \\
\hline \multicolumn{2}{|c|}{ - Distributed content name } \\
\hline \multicolumn{2}{|c|}{$\begin{array}{l}\text { - Description of the } \\
\text { distributed content }\end{array}$} \\
\hline \multicolumn{2}{|c|}{ - Date of registration } \\
\hline \multicolumn{2}{|l|}{ - Registrant ID } \\
\hline \multicolumn{2}{|l|}{ - Homepage } \\
\hline \multicolumn{2}{|l|}{ - Facebook } \\
\hline - Blog & \\
\hline$-\ldots$ & \\
\hline
\end{tabular}

\section{PPL attributes}

\section{Figure 7. An Example User nterface fon Creating Metadata}

\subsection{Database System}

A part of the tables included in gur database are.

1) ApprovedDistributionInfo consisting of DistributionApprovalID, contentGroupID, BusinessID, StartDate, ERdDate, Status, RegistrationDate, processID, processDate, processReason. An exâmple query to retrieve all approved distributions is as follows:

SELECT DistributionApprovalfid, contentGroupID, BusinessID, StartDate, EndDate, Status, RegistrationDate, processiD. processDate, processReason

FROM ApprovedDistríbutioninfo D;

2) transformInfo consistmg of DistributionApprovalID and transformType.

3) distributedContent consisting of distributedContentID, status, homepageURL, copyrightExpirationĐate, twitterURL, blogURL, me2dayURL, facebookURL, distributedContentName, EnglishName, deployID, distributedContentType, DistributionApprovalID, transformType, RegistrationDate, RegistrantID, modifyDate, modifierD.

4) distributionDeployHistory consisting of distributedContentID, deploySequenceNumber, deployType, requestType, registrationDate, deloyID.

5) userUsergroup consisting of userGroupID and userID.

6) userGroup consisting of userGroupID, userGroupName, deletedTF, description, registrationDate, registratioinID, modifyDate, modifyID.

7) menu consisting of menuID, upperMenuID, menuName, menuDescription, usingTF, linkURL, sortOrder.

8) authorizedGroupMenu consisting of userGroupID and menuID. 


\section{Implementation}

For management of distribution approval information, we implemented the classes shown in Figure 8. getList() retrieves a list of all distribution approvals. approve() approves the distributions designated by the parameter, appIds. deny() disapproves distributions designated by the appIds input parameter and records the reasons. getDstbApprDtl() retrieves detailed information of the distribution approval designated by the input parameter, dstbApprId. modifyDstbApprPeriod() modifies the period of distribution.

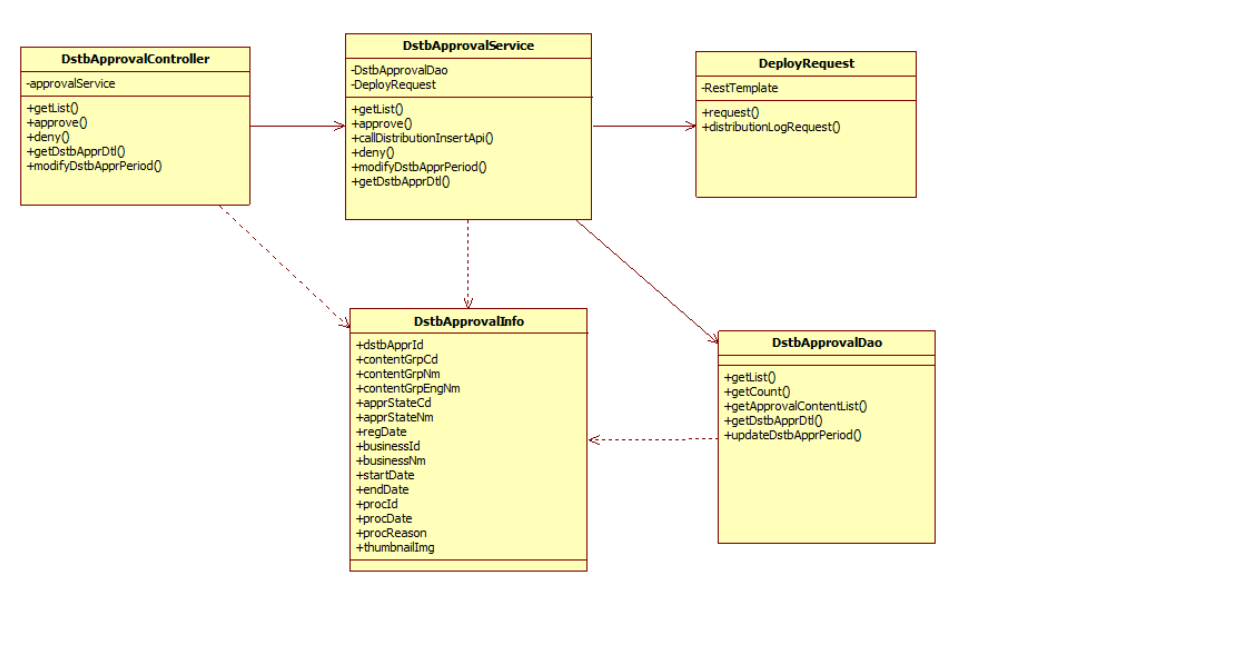

Figure 8. The Classes to Manage Distribution Approval Information

For retrieving deployment and content information, we implemented the classes shown in Figure 9. getDeployInfoDtl() returns detailed information of the deployment designated by input parameter, deployID. Simiary, getCrsContentDtl retrieves detailed information of the content designated by the input parameter, orgCntentId.

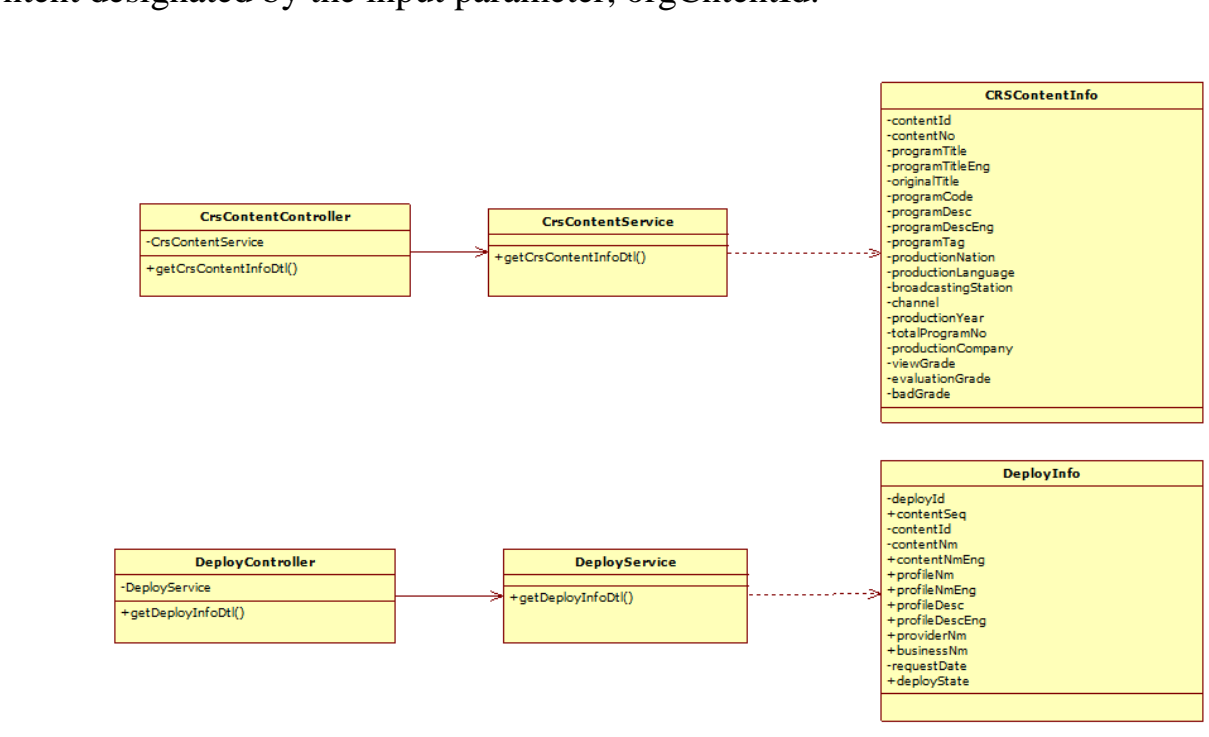

Figure 9. Classes to Retrieve Content and Deployment Information 


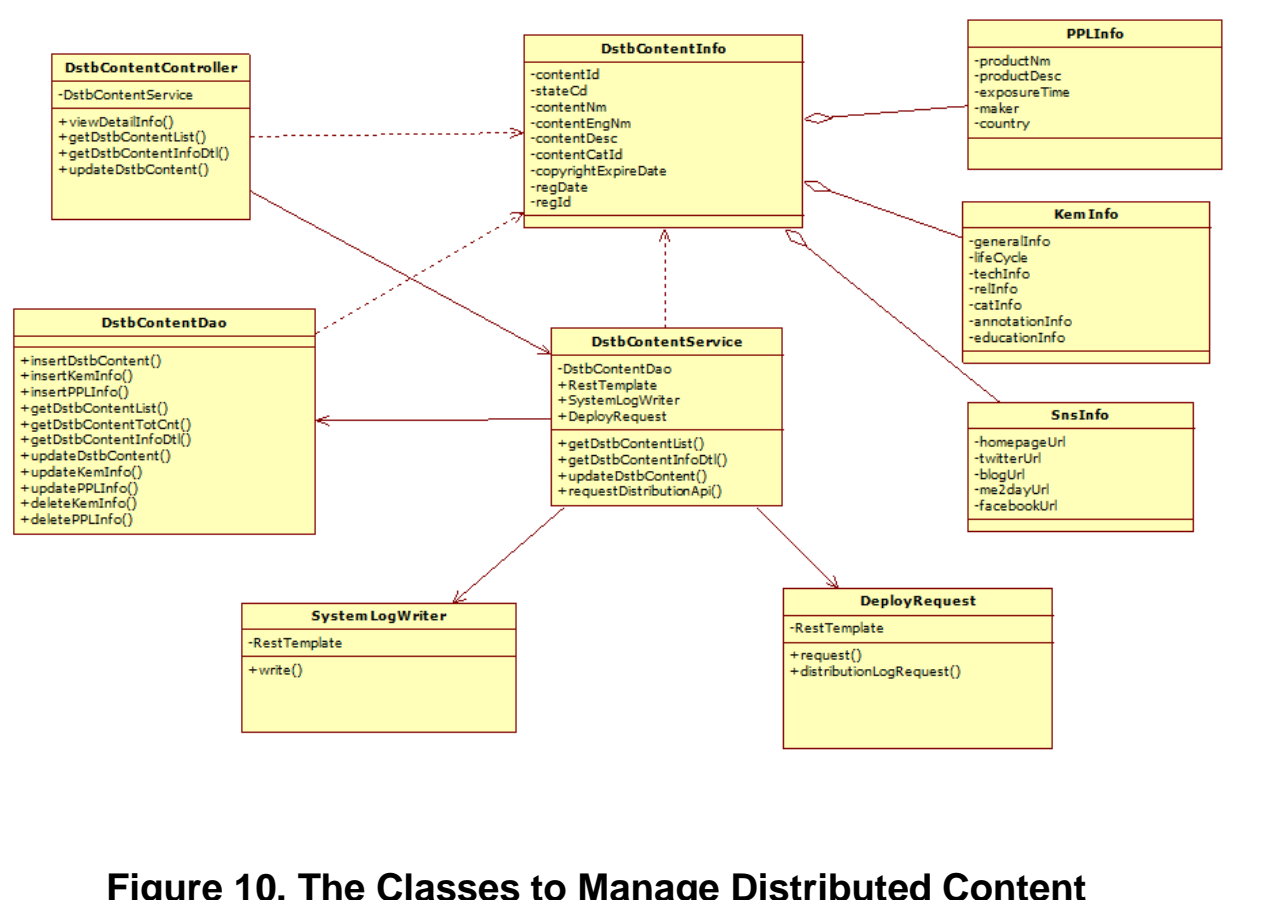

Figure 10. The Classes to Manage(Distributed Content

In order to retrieve and update ifformation of distributed content, we implemented the classes shown in Figure 10. getDstbContentList returns a list of all distributed content. getDstbContentInfoDtl() retrieves detailed information of the distributed content designated by the parameter dstrContentID. updateDstbContent() updates distribution content information with input parameters, EdudationMetadata, productName, productDescription, and so on.

In order to create retrieve, update and delete on-air information, we implemented the classes shown in Figure 16., getOnAirList returns a list of all information of on-air. insertOnAirInfo() takes OnAmrnfo parameter and insert it into the database.

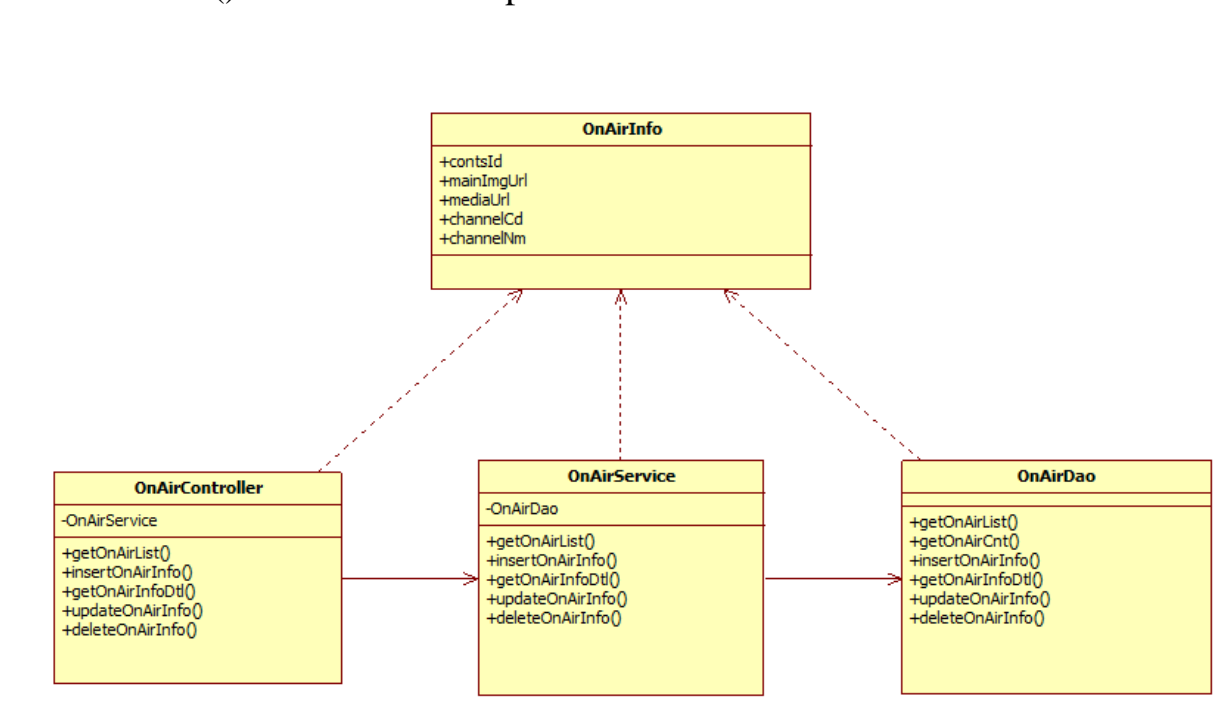

Figure 11. The Classes to Manage On-Air Information 


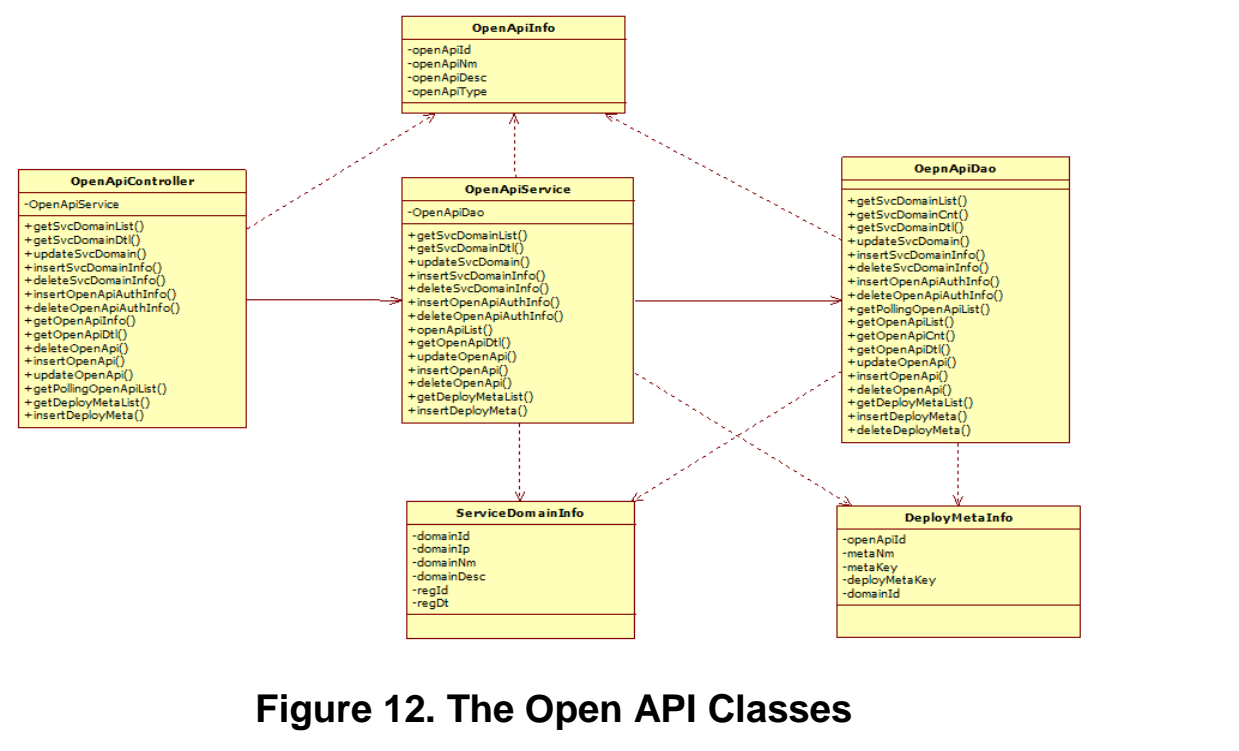

The distribution management system provides oper APIs shown in Figure 12. getSvcDomainList() returns a list of service domain information. getSvcDomainDtl() returns information of the service domain designated by the input parameter, domainID. getOpenApiInfo() returns a list of all open APIs. getOpenApiDtl() returns detailed information of the API designated by the input parameter, openApiId.

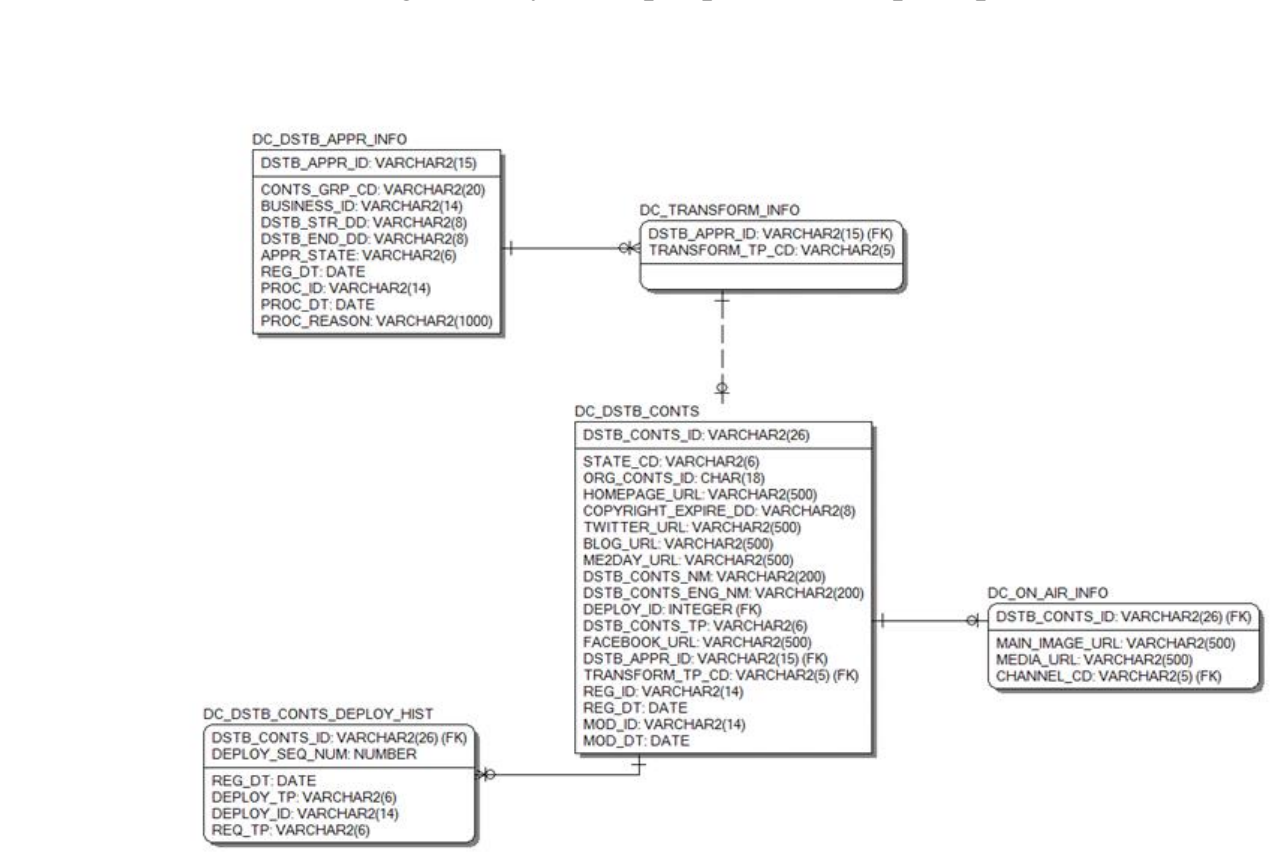

Figure 13. Our Database To Store Information about Distribution Approval 


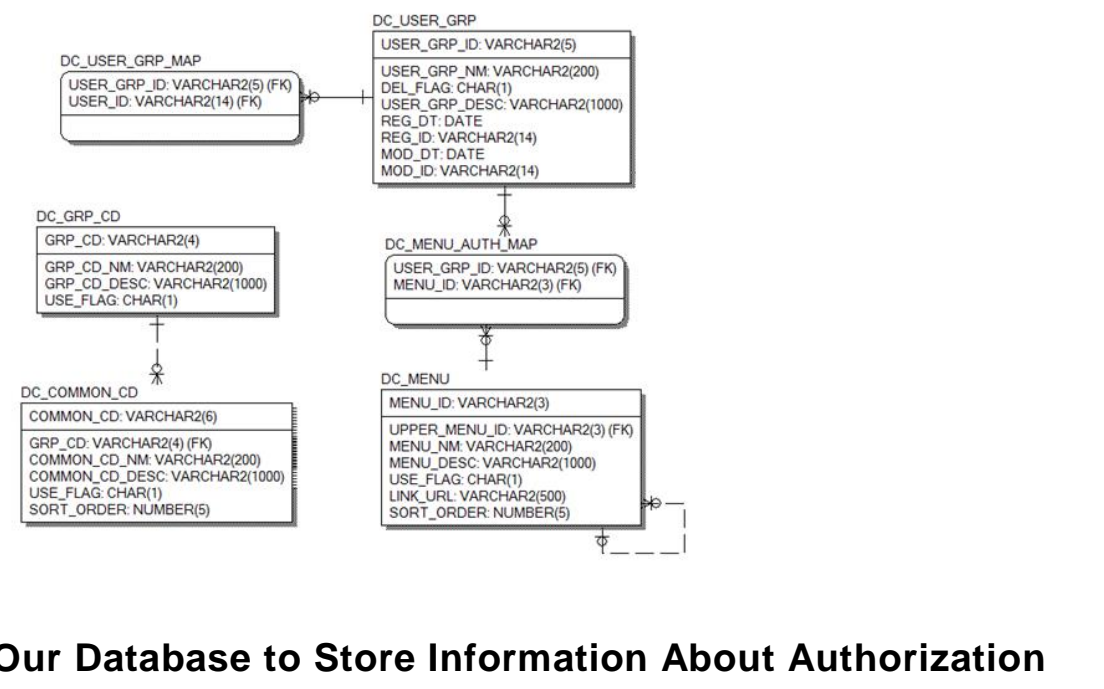

Figure 14. Our Database to Store Information Aboul Authorization

We implemented a database shown in Figure 13 in ofaer to store information about distribution approvals. DC_DSTB_APPR_NNFO represents etistribution approval information. A distribution approval requests for transcoding original content into the format required by the distributor. A distributor may asks for several content formats for smartphone, PC, tablet $\mathrm{PC}$, and so on.

We implemented another database in orderoto store information about authorization as shown in Figure 14. Many users belong to a user group, but a user can belong to exactly one user group. A user group can, be authorized to perform many menu items.

\section{Experiments}

We performed experiments of testing our system. An example screenshot of approving a purchase order is shown in Figure 15. A list of purchase orders is displayed in the screen. System operators can sefect one or more orders to approve or reject.

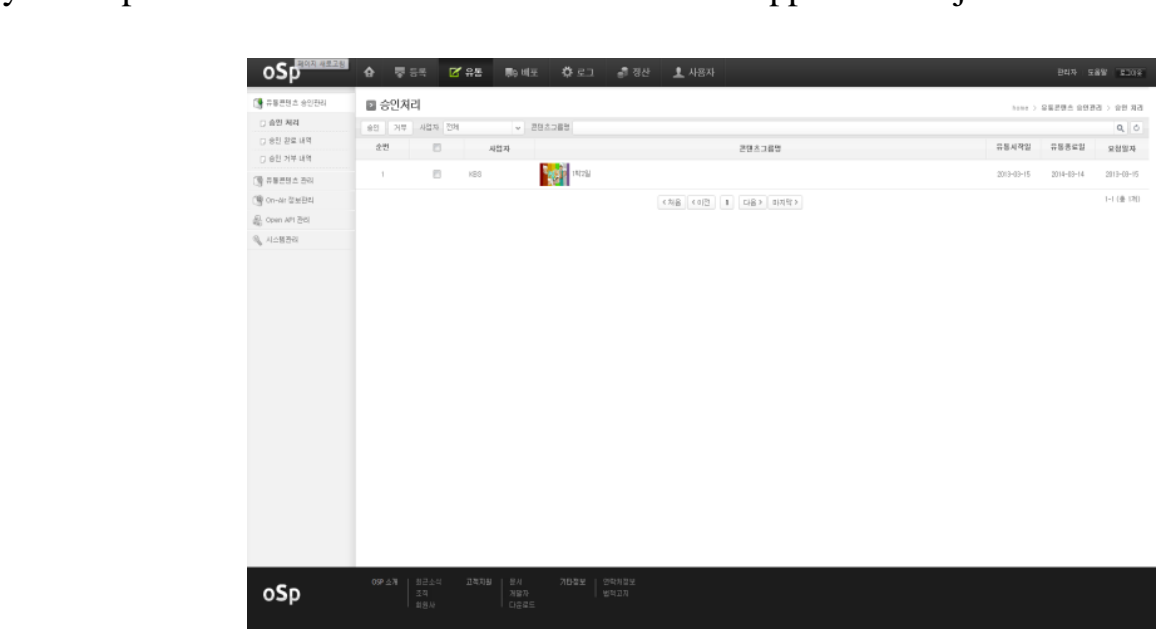

Figure 15. An Example Screenshot of retrieving a List of Purchase Orders to Approve 
Distribution management system allows system managers to retrieve distribution IDs and to type in detailed metadata of a distribution. A screenshot of the first step of typing in detailed metadata of a distribution is shown in Figure 16.

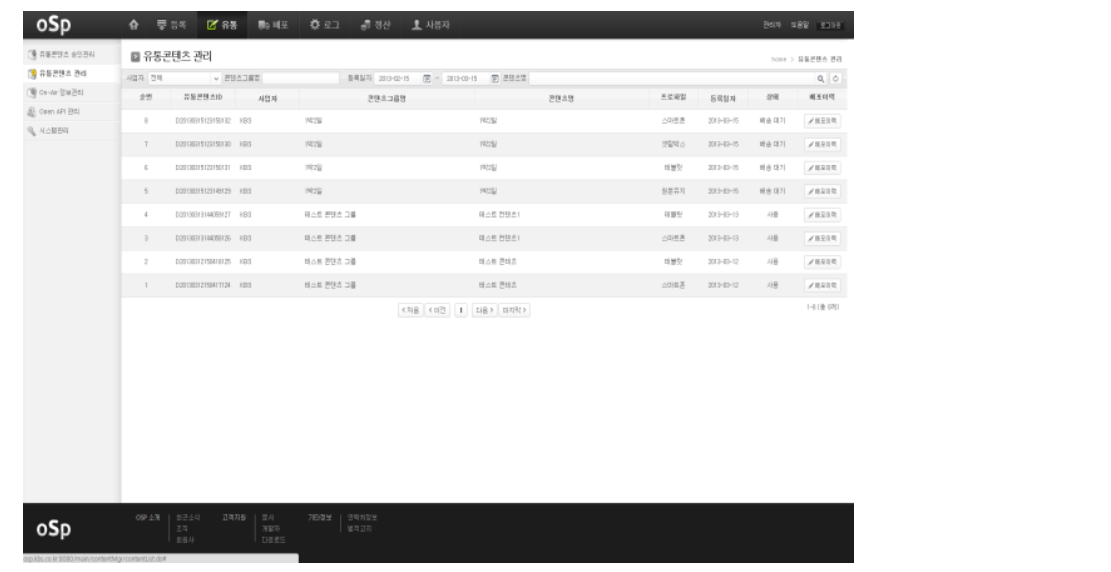

\section{Figure 16. A Screenshot of Retrieving Distribution IDs}

\section{Conclusions}

The distribution management system of an IPTV system allows distributors to request for content circulation and system mánagers to process distributors' requests. Therefore, we can notice that a distribution management system is one of the key components for realizing one source multi-use. Our system will contribute to boost circulating value-added content and to boost the content industry.

\section{Acknowledgments}

This work was sxpported by Basic Science Research Program through the National Research Foundation of KKea(NRF) funded by the Ministry of Education (NRF-20110006942) and by 'Development of Global Culture and Tourism IPTV Broadcasting Station' Project through the Industrial Infrastructure Program for Fundamental Technologies funded by the Ministry of Khowledge Economy (10037393).

This paper is a revised and expanded version of a paper entitled "Introducing an IPTV Platform Design" presented at ACIT 2014, Michigan, USA, July 9-12, 2014.

\section{References}

[1] http.//en.wikipedia.org/wiki/IPTV

[2] J. Yim and G. Lee. The Design and Implementation of a Broadcasting Management Web System for IPTV. International Journal of Multimedia and Ubiquitous Engineering 8, (2013)

[3] www.zooin.net

[4] X. Hei, C. Liang, J. Liang, Y. Liu, K. Ross. A Measurement Study of a Large-Scale P2P IPTV System. IEEE TRANSACTIONS ON MULTIMEDIA, VOL. 9, NO. 8, DECEMBER 2007, pp.1672-1687

[5] T. Qiu, Z. Ge, S. Lee, J. Wang, J. Xu, Q. Zhao, Modeling user activities in a large IPTV system, Proceedings of the 9th ACM SIGCOMM conference on Internet measurement conference Pages 430-441

[6] J. Yim and G. Lee. Introducing an IPTV Platform Design. Proceedings of the 2nd International Conference on Advanced Computer and Information Technology (2014) July 9-12; Oakland University, Michigan, USA

[7] J. Yim, G. Lee, T. Le, "Design and Implementation of VOD Database System," IJMUE Vol.7, No.2, 503-508 (2012)

[8] J. Yim, G. Lee, "Minimum Cycle Time Analysis of IPTV Systems," IJMUE Vol.7, No.2, 119-134 (2012) 
[9] M. Díaz, E. Huh, "Secure Collaboration Mechanism for SLA delivery among IPTV providers," IJCA Vol. 4, No. 1, 71-85 (2010)

[10] D. Seo, B. Kim, H. Lee, S. Seo, "Virtual Channel Management for IPTV using Channel Domain Systems," IJCA Vol. 5, No. 2, 65-72 (2012)

[11] S. Kim, B. Park, "SM-IPTV: A Research on Optimized Handover Routing Architecture for Seamless Multimedia Convergence Service over Wireless Networks," IJCA Vol. 5, No. 3, 249-258 (2012)

\section{Authors}

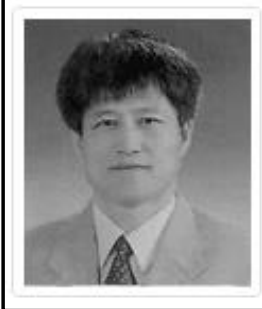

Jaegeol Yim received the M.S. and Ph.D. degrees in Computer Science from the University of Illinois at Chicago, in 1987 and 1990, respectively. He is a Professor in the Department of Computer Science at Dongguk University at Gyeongju Korea. His/current research interests include Petri net theory and its applications, Location Based Service, AI systems and multimedia systems. He has published more than 50 journal papers, 100 conference papers (mostly written in Korean Language), and several undergraduate textbooks.

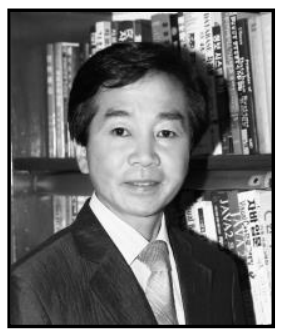

Gyeyoung Lee, received the M.S.Adegree in Computer Science from the Dongguk University in 1982 and the Ph.D. degree in Computer Engineering from the Dankook University in Korea in 1992, respectively. $\mathrm{He}$ is a Professor in the Defartment of Computer Science at Dongguk University at Gyeongju, Korea. His current research interests include Petri net theory, AI systemsand Speech processing systems.

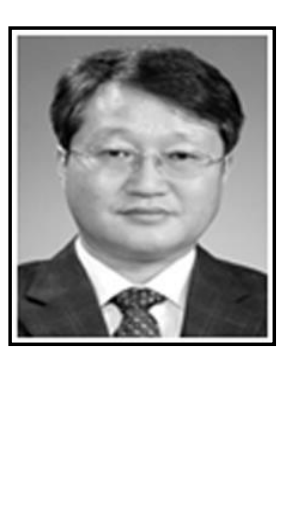

Kyubark Shin received the M.S. and the Ph.D. degrees in Statistics from the Dongguk University in 1986 and 1993, respectively. He is a Professo in the Department of Information and Statistics at Dongguk University at Gyeongju, Korea. His current research interests include Computational Statistics, Reliability Test, Analysis of Statistical Data. 